\title{
Research Paper: Investigating the Relationship Between Selective Attention and Cogni- tive Flexibility With Balance in Patients With Relapsing-Remitting Multiple Sclerosis
}

\author{
Vali Shiri, ${ }^{1,2}$, Mahbubeh Emami ${ }^{3}$, Esmail Shiri ${ }^{4}$
}

1. Department of Occupational Therapy, School of Rehabilitation Sciences, Isfahan University of Medical Sciences, Isfahan, Iran.

2. Musculoskeletal Research Center, Isfahan University of Medical Sciences, Isfahan, Iran.

3. Department of Occupational Therapy, University of Social Welfare and Rehabilitation Sciences, Tehran, Iran.

4. Department of Clinical and Health Psychology, Faculty of Psychology and Educational Sciences, Shahid Beheshti University of Medical Sciences, Tehran, Iran

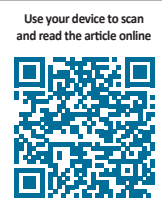

Citation: Shiri V, Emami M, Shiri E. [Investigating the Relationship Between Selective Attention and Cognitive Flexibility With Balance in Patients With Relapsing-Remitting Multiple Sclerosis (Persian)]. Archives of Rehabilitation. 2018; 18(4):296-305. https:// doi.org/10.21859/JREHAB.18.4.4

\section{https://doi.org/10.21859/JREHAB.18.4.4}

Received: 27 Aug. 2017 Accepted: 17 Nov. 2017

Keywords:

MS, Selective attention, Cognitive flexibility, Balance

\section{A B STRACT}

Objective Multiple Sclerosis (MS) is known as one of the most common neurological disorders that affect patients' lives tremendously. These people experience various dysfunctions. One of the most common problems in patients with MS is a balance deficit, which limits the participation and increases the risk of falling. Recently various studies were focused on finding the causes of this imbalance. On the other hand, these patients experience cognitive problems, such as attention deficit and cognitive flexibility. Therefore, the aim of this study was to investigate the relationship between cognitive flexibility and selective attention with balance in patients with relapsing-remitting MS.

Materials \& Methods This study is a cross-sectional case study. The statistical population in this study was patients with MS who referred to Kashani Hospital and Alzahra Isfahan Hospital in 1395 for inclusion in the study, based on available sampling method. MS diagnosis was performed in these centers with the approval of at least one adult neurologist. Finally, 40 patients with MS were selected by convenient sampling. A written consent form was received from all the families and the patients with MS themselves for participation in the study. All patients were kept confidential in the study, and they were allowed to leave during the study if they refused. The inclusion criteria to participate in the study were: satisfaction of patients to participate in the plan, the individual received a diagnosis of relapsing-remitting MS from those who were referred to Al-Zahra and Kashani hospitals in Isfahan, aged 20 to 40 years, at least two attacks in last 24 months, a score of less than 5.5 in EDSS was associated with a moderate degree of MS due to the severity of the disability and the symptoms of the disease, having sufficient insight and the ability to collaborate in the study through the MMSE test. The exclusion criteria of the study were: dissatisfaction of the patients with the continuation of the study, presence and participation in similar investigations in the last three months that were performed intermittently on executive functions, the presence of visual and auditory impairment, recurrence and severity of MS, disability from moderate to severe, and any abnormalities such as seizure. After that test EDSS, Stroop color-word test, Wisconsin card sorting, and Berg Balance Scale were completed by patients with MS. The results of the tests of executive function and balance were analyzed using SPSS20 software for the presence or absence of the relationship between executive function is determined by the balance. To analyze the data, Pearson correlation coefficient and multiple regression and analysis of variance with repeated measures at the level of 0.05 .

Results The results of this study demonstrate a significant relationship between selective attention and balance problem $(\mathrm{P}<0.05)$. However, there is no significant relationship between cognitive flexibility and balance $(P<0.05)$. As well as the selective attention can predict the balance problem $(P<0.05)(P<0.01)$.

Conclusion A significant relationship between selective attentions to balance demonstrates that appropriately designed interventions to repair the attention, can decrease the balance problem in patients with MS. One of the possible reasons for this relationship can be found in the brain system that attention circuits are interacting with equilibrium systems, and any defect in selective attention leads to a loss of balance. Patients with MS experience constant imbalance due to cerebral plaques and relapsing-remitting periods, and in addition to rehabilitation of the balance system, cognitive systems such as attention should be treated.

\section{* Corresponding Author:}

Esmail Shiri, PhD Candidate

Address: Department of Clinical and Health Psychology, Faculty of Psychology and Educational Sciences, Shahid Beheshti University of Medical Sciences, Tehran, Iran.

Tel: +98 (912) 9328865

E-Mail: esmaeil.shiri67@yahoo.com 


\title{
بررسى رابطه بين توجه و انعطافيذيرى شناختى با تعادل در بيماران اماس عود - فروكش
}

\author{
ولى شيرى"، محبوبه امامى"، "اسماعيل شيرى"

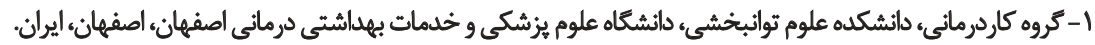

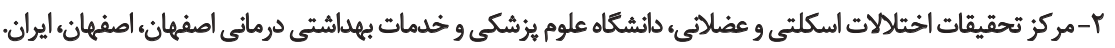

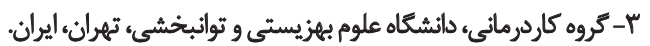

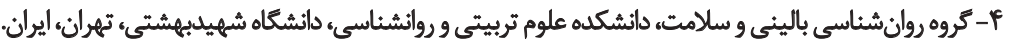

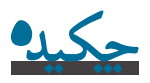

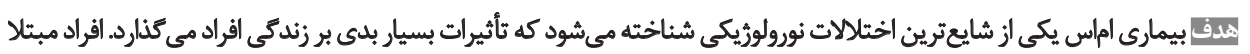

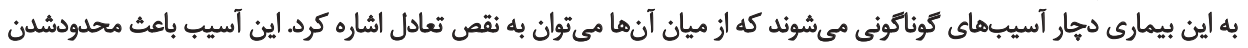

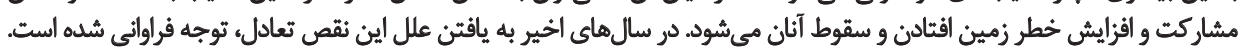

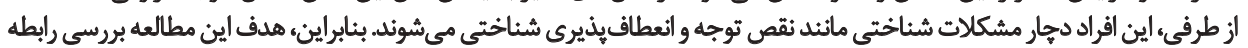

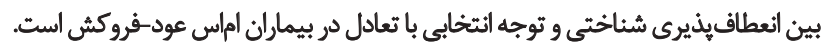

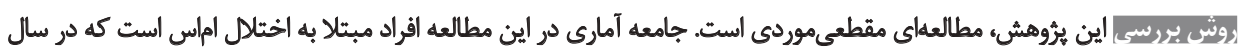

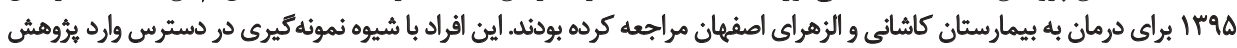

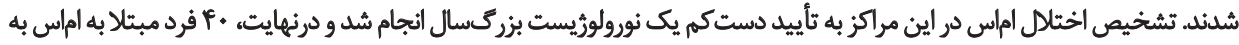

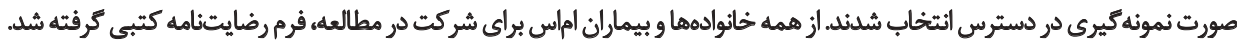

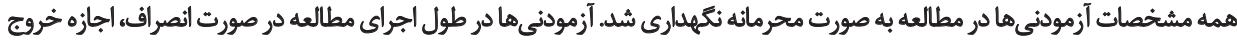

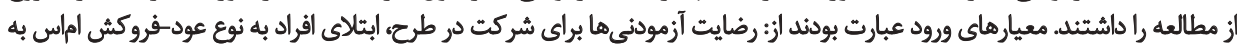

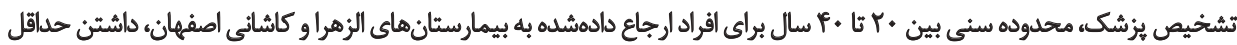

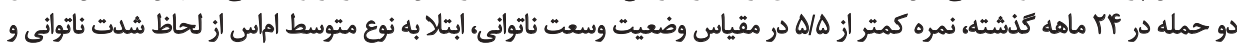

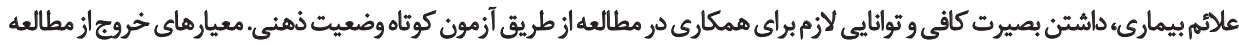

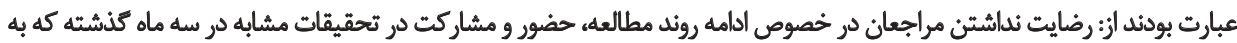

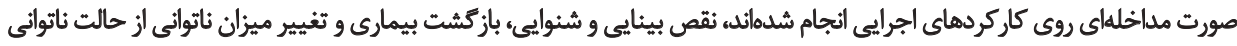

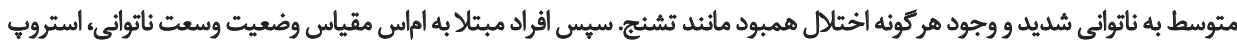

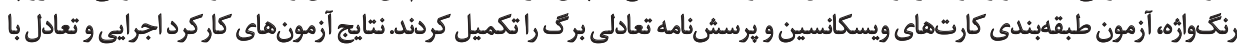

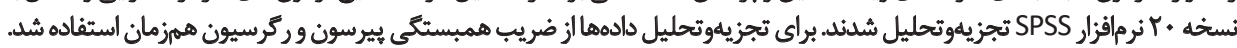

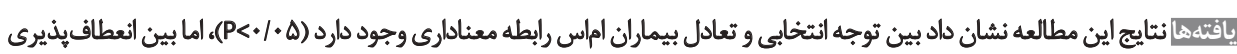

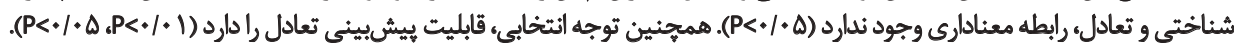

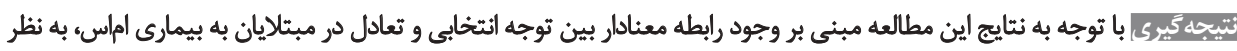

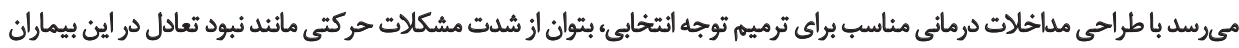

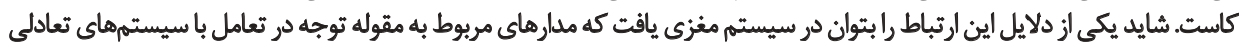

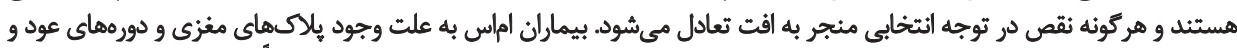

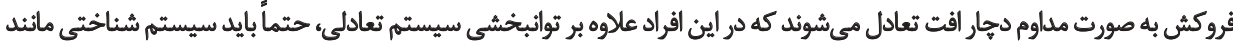

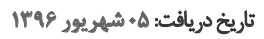

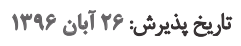




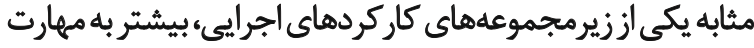
مقلمه

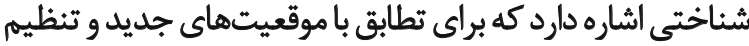

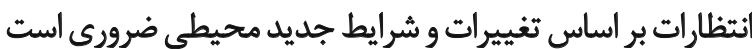

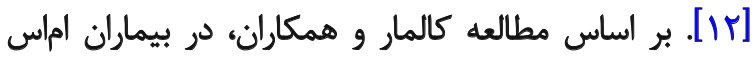

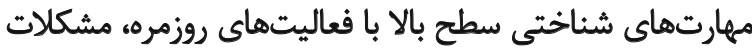

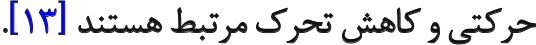

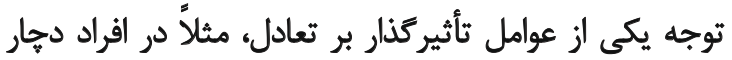

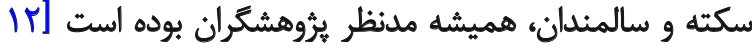

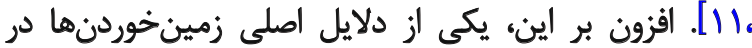

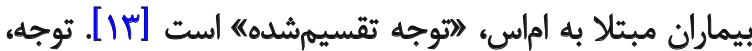

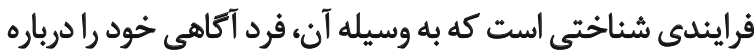
محركهاى كشفشده به وسيله حواس، هدايت و و حفظ مي كئد

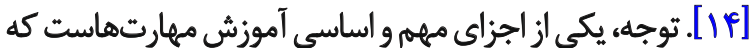

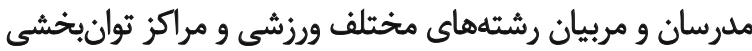

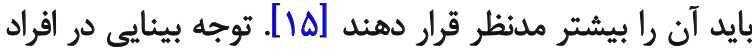

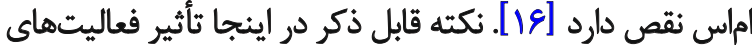

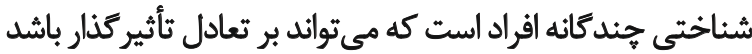

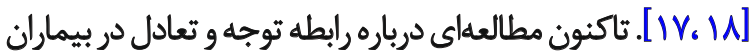

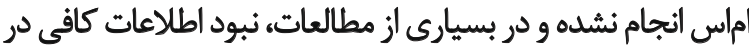

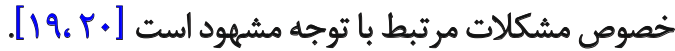

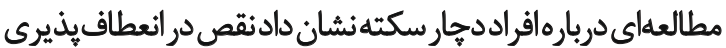

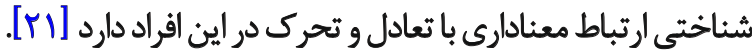

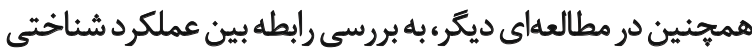

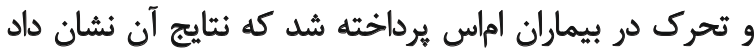

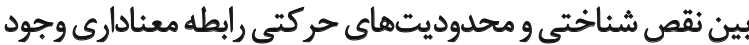

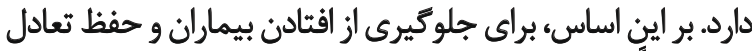

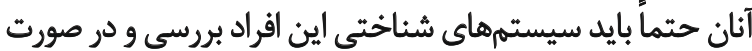

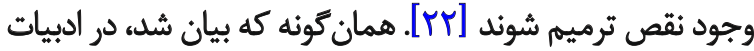

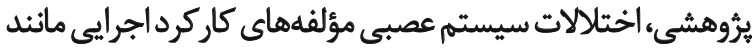

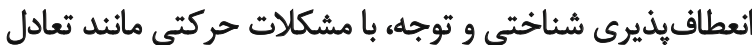

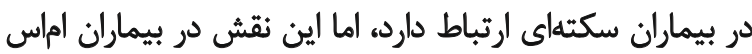

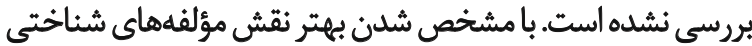

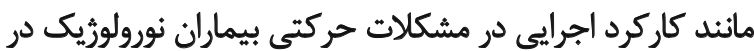

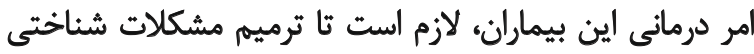

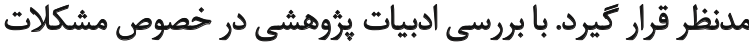

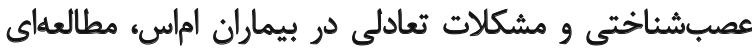

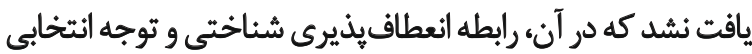

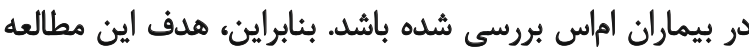

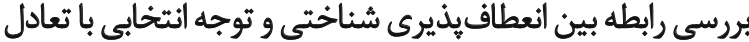

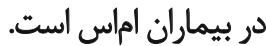

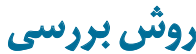
اين يُروهش، مطالعهاى مقطعى موردى است. جامعه آمارى در

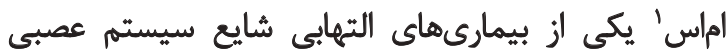

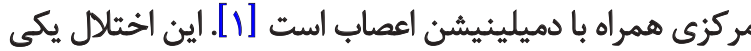

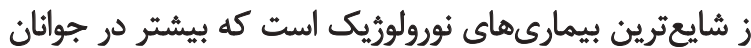

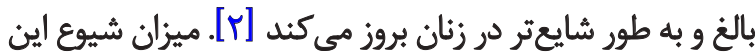

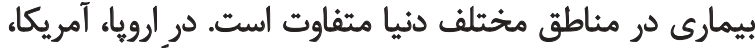

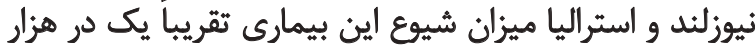

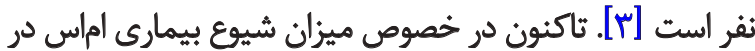

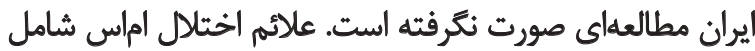

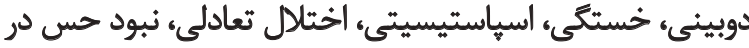

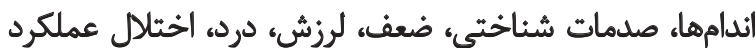

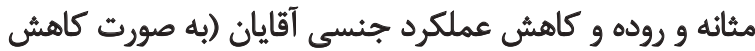

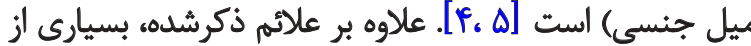

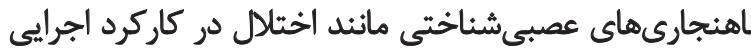

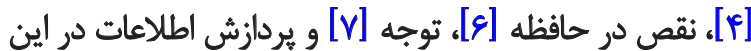

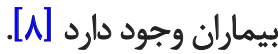
بيماران اماس دجار اختلالات اسكلتى عضلانى، عصبى و تعادلى إنى

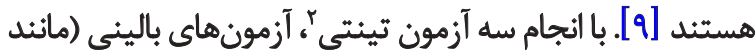

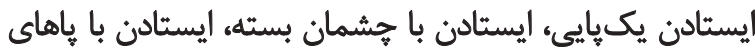

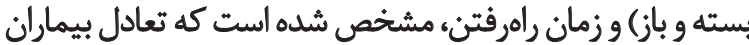

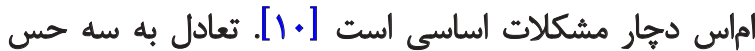

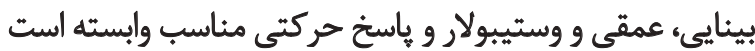

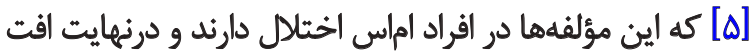

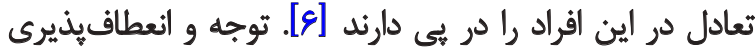

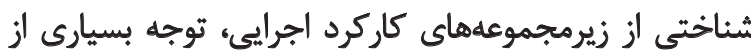

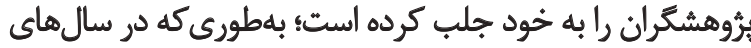

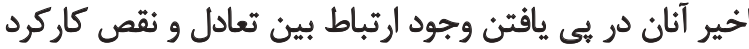

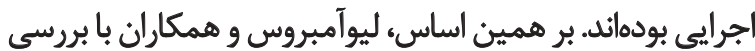

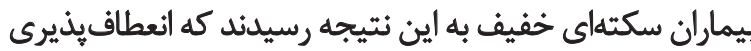

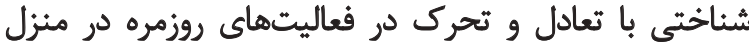

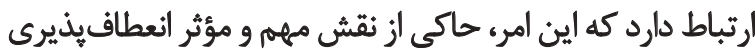

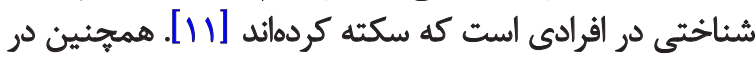

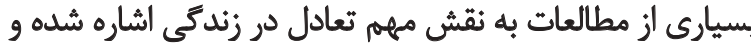

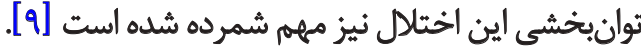

كاركرد اجرايى در واقع ساختارى شناختى است كه از اين ين ئنادي

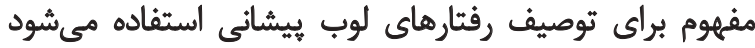

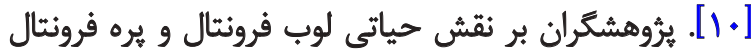

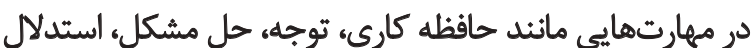

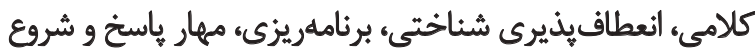

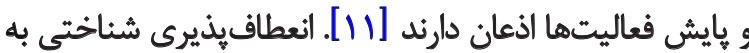




\section{آزمون رنك-وارهه استروب}

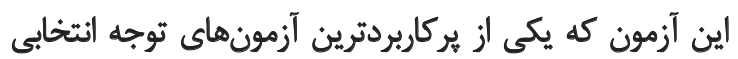

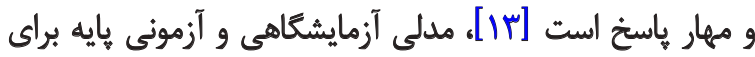

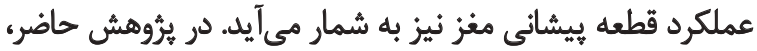

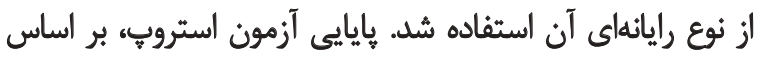

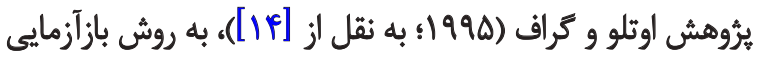

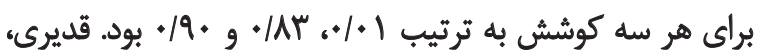

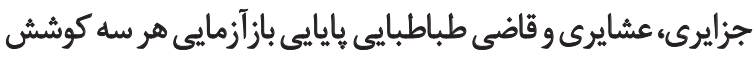

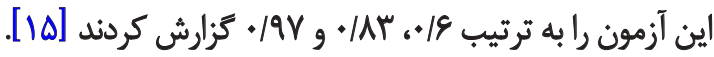

\section{آزمون طبقهبندى كارتهاى ويسكانسين}

از اين آزمون براى ارزيابى مهارت انعطافيذيرى شناختى،

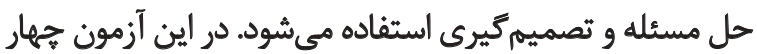

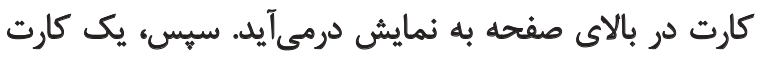

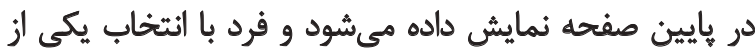

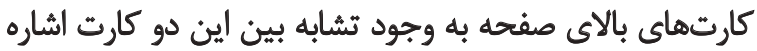

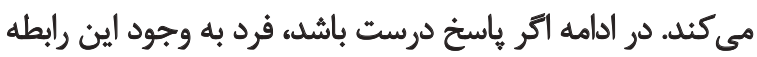

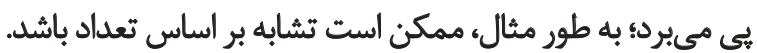

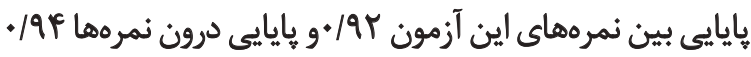

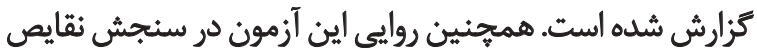

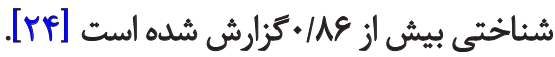

\section{يرسش ئامه تعادلى برى}

اين مقياس شامل \& ا آيتم (فعاليتهاى متداول زندكى روزانه)

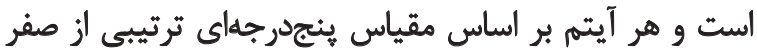

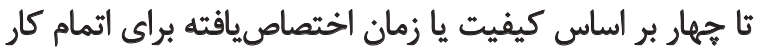

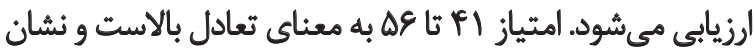

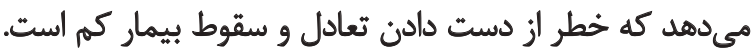

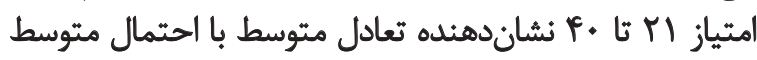

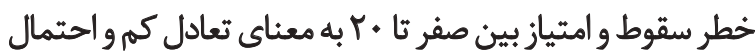

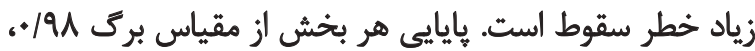

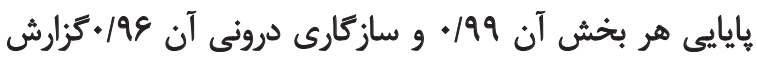

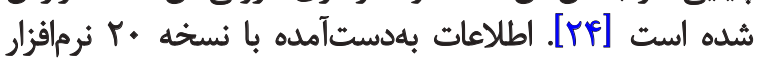

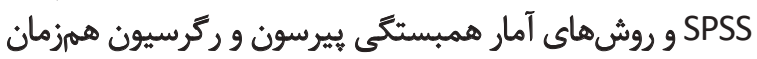

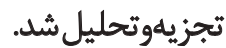

يافتهها

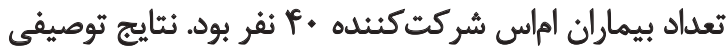

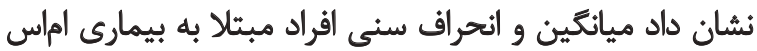

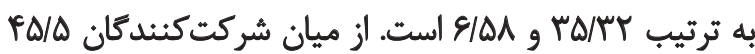

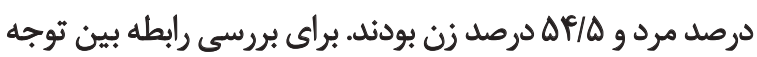

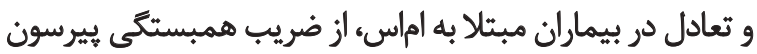

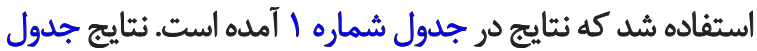

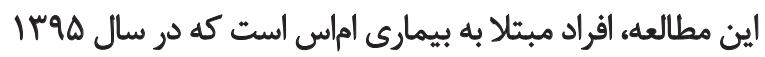

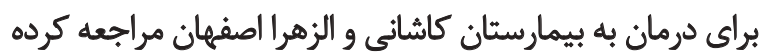

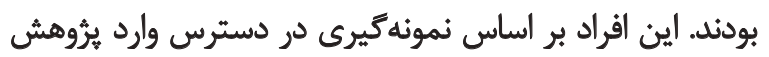

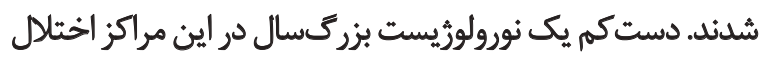

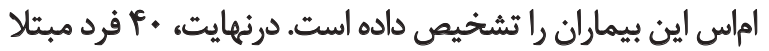

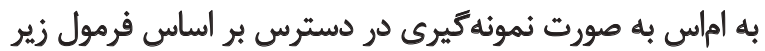

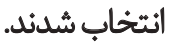

$$
n=\left[\frac{Z_{1-a / 2}+Z_{1-b}}{\frac{1}{2} \ln \left[\frac{1+r}{1-r}\right]}\right]^{2}+3
$$

از همه خانوادهها و بيماران اماس براي شركت در مطالعها

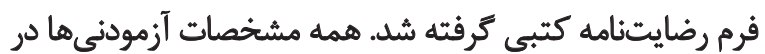

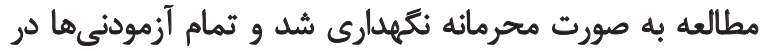

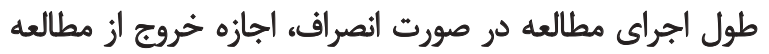

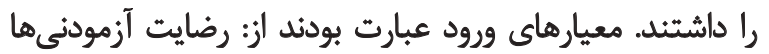

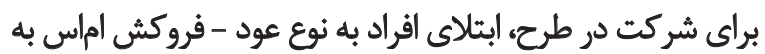

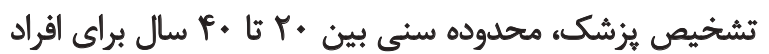

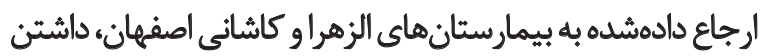

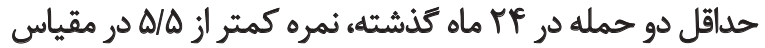

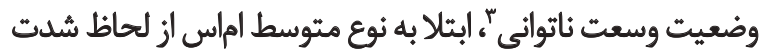

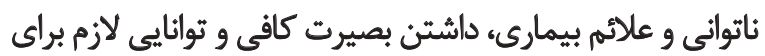

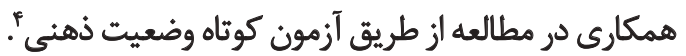
معيارهاى خروج از مطالعه عبارت بودند از: رضايت نداشتن

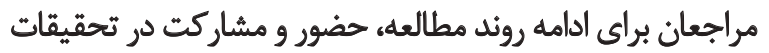

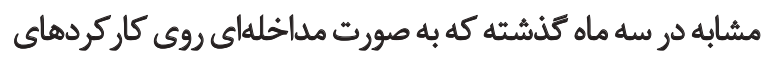

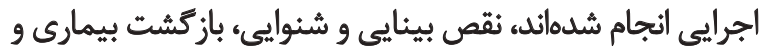

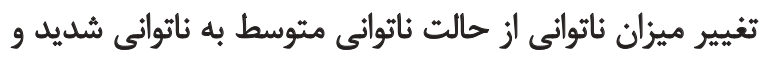

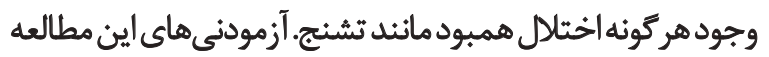

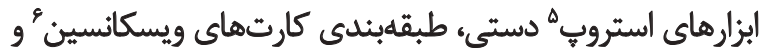

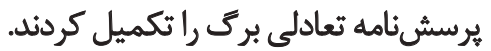

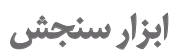

\section{مقياس وضعيت وسعت ناتوانى}

اين آزمون تغييريافته آزمون مقياس وضعيت وسعت ناتواني

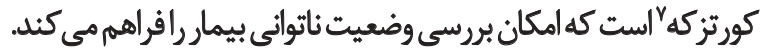

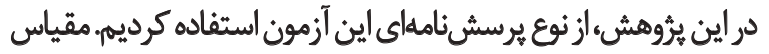

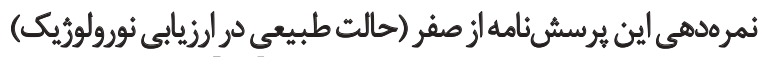

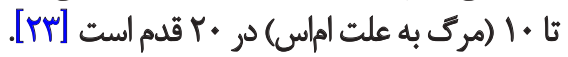

3. Expanded Disability Status Scale (EDSS)

4. Mini-Mental State Examination (MMSE)

5. Stroop

6.Wisconsin card sorting

7. Kurtzke 
جدول ا. ئتايج ضرايب همبستّكى بيرسون بين توجه و تعادل

\begin{tabular}{|cccccccccccc}
\hline & & & & & \\
\hline
\end{tabular}

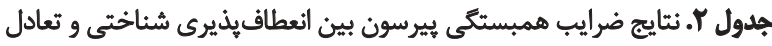

\begin{tabular}{|c|c|c|c|c|c|c|}
\hline \multicolumn{2}{|c|}{ ثعداد ياسخغاي صحيح } & \multicolumn{2}{|c|}{ خطاي درجامانديمي } & \multicolumn{2}{|c|}{ تعداد طبقات } & \multirow{2}{*}{ مثغير } \\
\hline P Pرش P & Rقدار R R & P ارزش P & R مقدار R & P ارزش P & Rقدار R & \\
\hline . IAE & || درصد & - IEAP & آM درصد &.$/ M T$ & ها درهد & تعادل \\
\hline
\end{tabular}

جدول ".. خلاصه اطلاعات جدول تحليل واريائس

\begin{tabular}{|c|c|c|c|c|c|c|c|c|}
\hline$R^{r}$ & $\mathbf{R}$ & $P$ & $\mathbf{F}$ & مجذياتينت & أزادي & جمع مجذورات & شاخص آمارى & مدل \\
\hline \multirow[t]{3}{*}{.19.} &.$/ 9 \Delta$ & 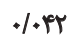 & Q/TTA & rur/qq & 9 & $r \Delta \Delta \Delta / q$ & ركرسيون & \\
\hline & & & & $\Delta \varphi / M$ & $\Delta$ & $m / W$ & باقى مائده & كلى \\
\hline & & & & & if & TATYMT & كل & \\
\hline
\end{tabular}

توانبخننى

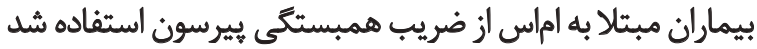

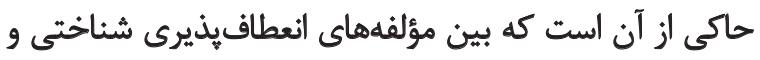
تعادل هيج رابطه معنادارى وجود ندارد اسن مؤه

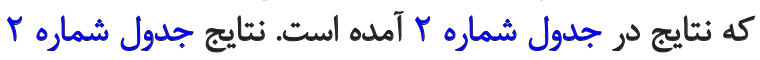

شيماره ا حاكي از آن است كه بين تعداد باستخهاي صحيح هر حله

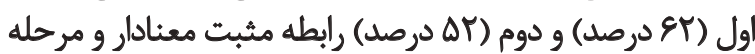

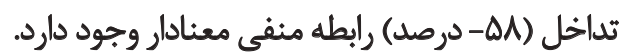
براى بررسي رابطه بين انعطافيذيرى شناختى و تعادل در

جدول عُ يُيشبينى تعادل از روى شاخص توجه و انعطاف بذيرى شناختى

\begin{tabular}{|c|c|c|c|c|}
\hline $\mathbf{P}$ & $\mathbf{T}$ & بتّا & متغير & مدل \\
\hline$\cdot / m n^{5}$ & -IANT & $-1 \mathrm{NOV}$ & تعداد ياسخ هاى صحيح مرحله اول & \\
\hline.$/ 4$ & - T/quq & $-/ A Y A$ & زمان واكنش مرحله اول & \\
\hline.$/ M$ & V/ATY & $1 / A 11$ & تعداد ياستخ هاي صحيح مرحله دوم & \\
\hline.$/ 49$ & $-M / 199$ & IgAt & تعلاد باسخخهاى صحيح مرحله سوم & \\
\hline.$/ 4 \mathrm{pr}$ & T/RT &.$M E$ & زمان واكنش مرحله سوم & كلى \\
\hline .1 .11 & $-r / u$. & -19.4 & ت تدائل & \\
\hline .119 & $-I M A$ & $-1 \cdot v$ & تعداد طبقات & \\
\hline.$|8 \wedge|$ & -1 tres & -1118 & خطاى درجاماندكى & \\
\hline.$/ 094$ & $. / \Delta V \mid$ &.$/ N F$ & تعداد ياسخحهاى صحيح & \\
\hline
\end{tabular}


اين امر مىتواند تأييدكنيده وجود رابطه بين اين دو مفهوم

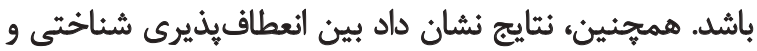

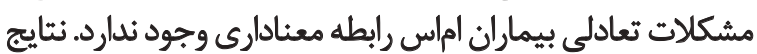

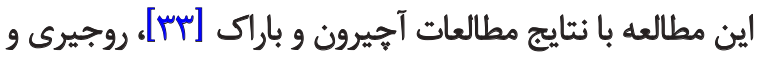

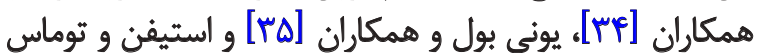

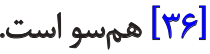

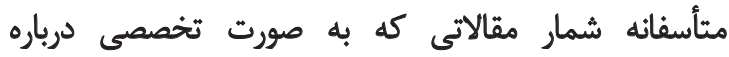

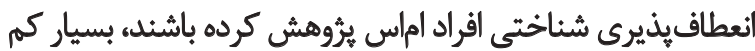

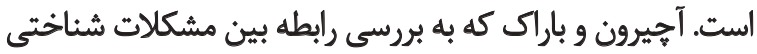

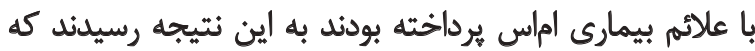

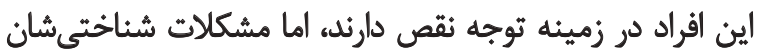

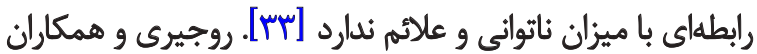

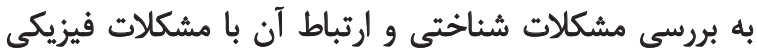

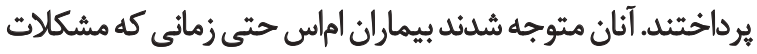

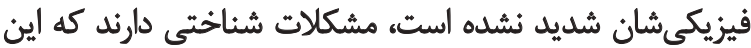

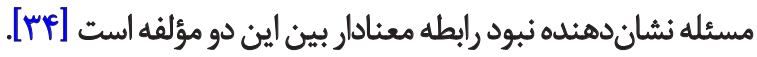

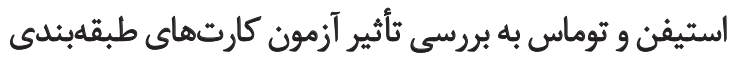

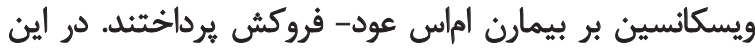

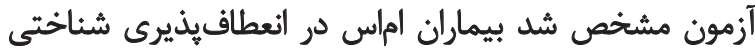

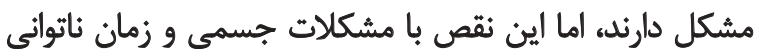

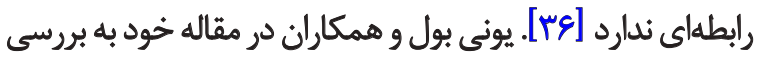

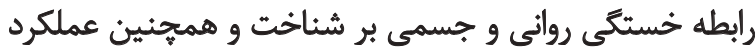

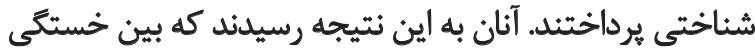

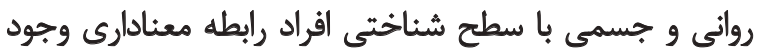

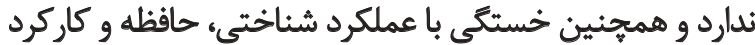

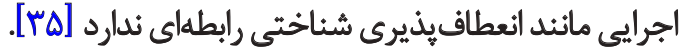

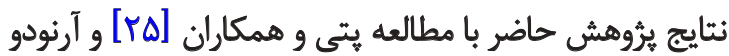

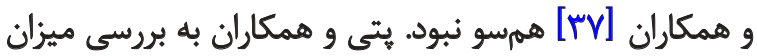

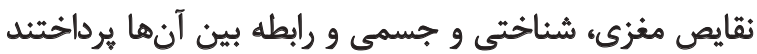

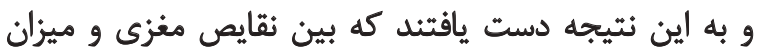

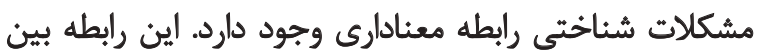

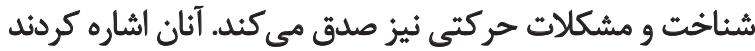

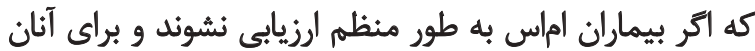

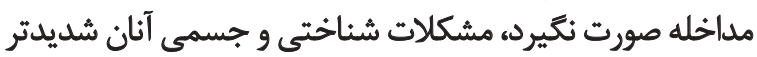

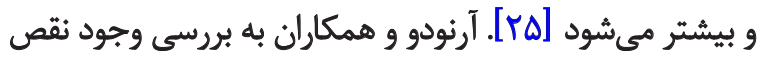

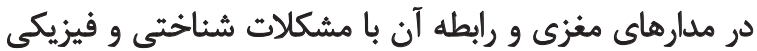

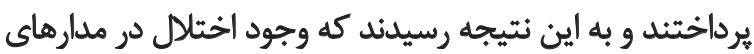

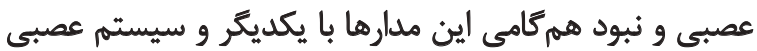

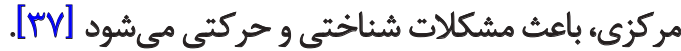
اين نتايج ناهمسو ميتواند ناشى از استفاده از آزمونهاي

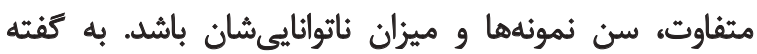
آجيرون و باراك، نتايج در حوزه شناختى تفاوت بنانيان بانيارى دارد

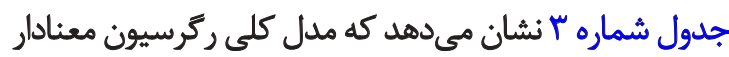

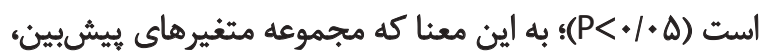

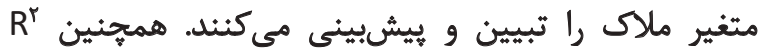

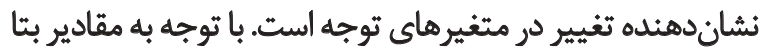

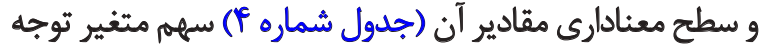

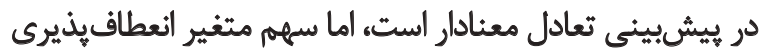

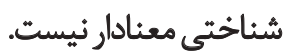
بحث

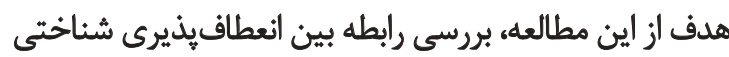

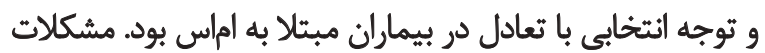

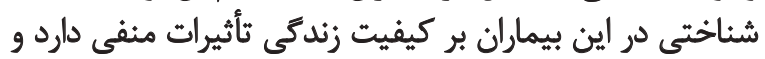

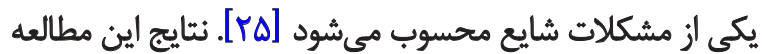

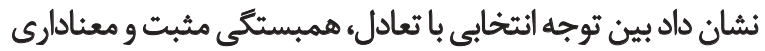

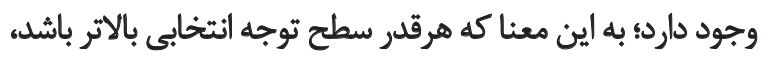

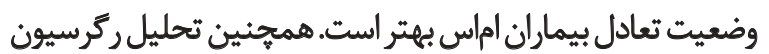

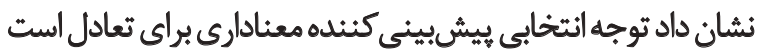

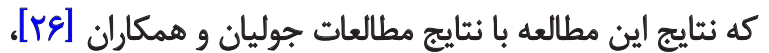

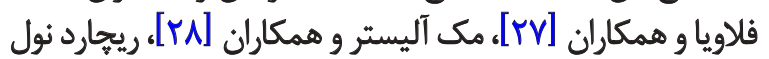

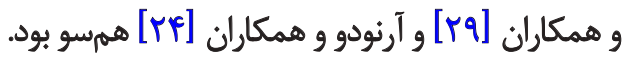
جوليان و همكاران رابطه شناخت با علائم بالينى افراد مبتلابه

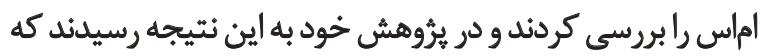

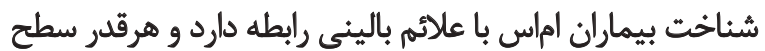

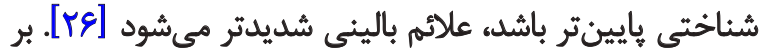

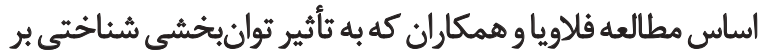

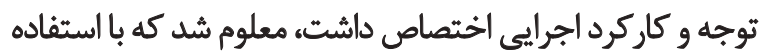

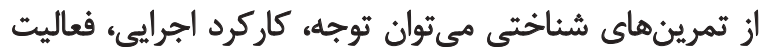

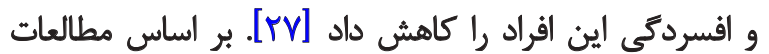

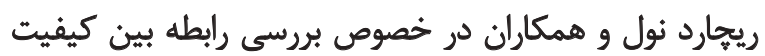

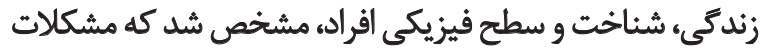

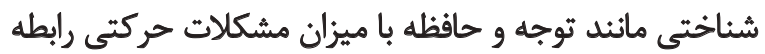

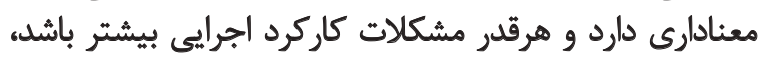

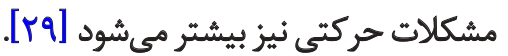

به نظر ميرسد يكى از دلايل اصلى وجود رابطه معنادار بين

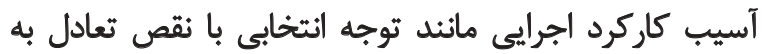

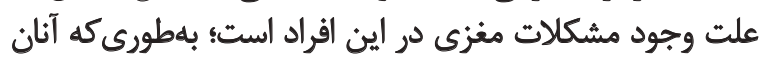

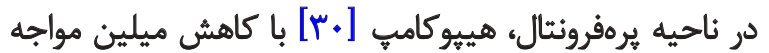

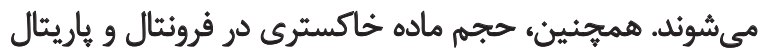

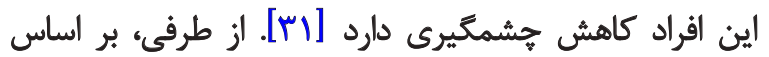

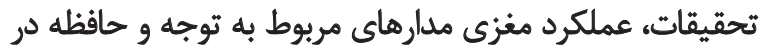

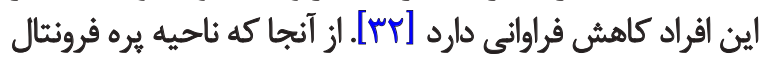

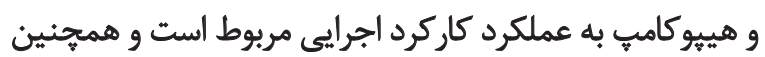

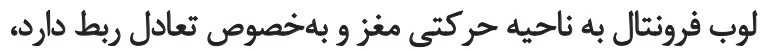


و اين تفاوت به علت اسثفاده از آزمونهاى متفاوت و نمونههاى

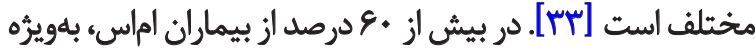

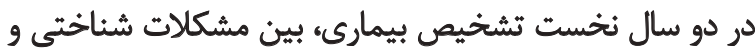

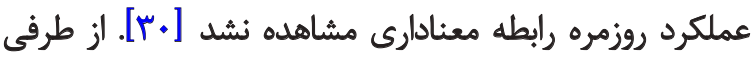

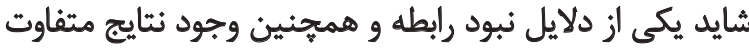

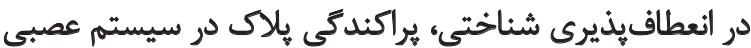

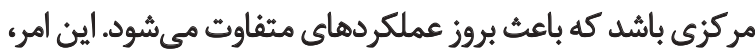

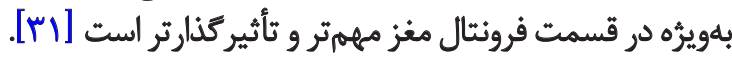

$$
\text { تثيجلمَيرى }
$$

با توجه به نتايج اين مطالعه مبنى بر وجود رابطه معنادار بين

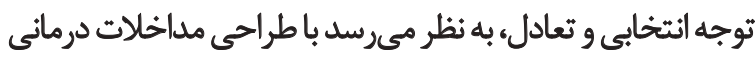

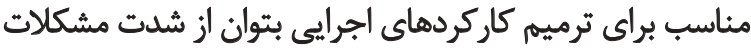

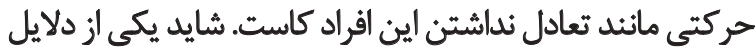

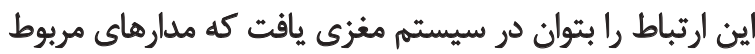

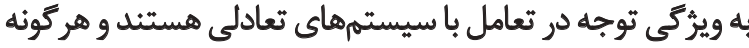

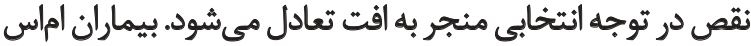

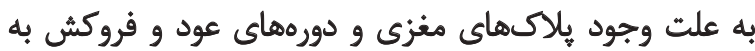

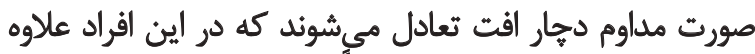

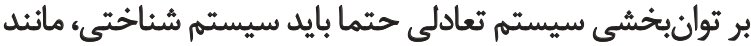

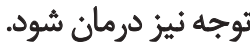

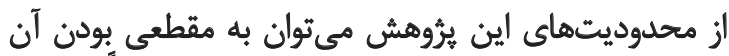

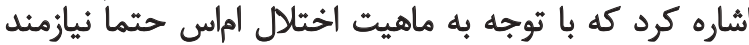

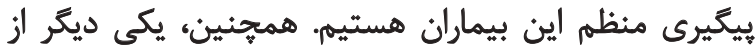

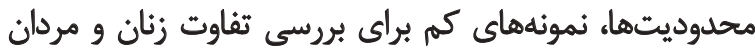

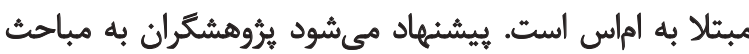

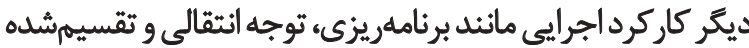

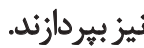

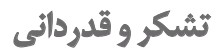

از تمام افراد مبتلا به اماس كه در اين مطالعه شركت كردند

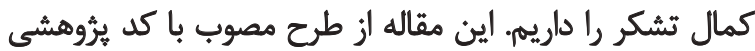
تحقيقات اختو و كد اخلاقى

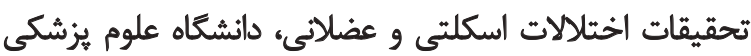
اصفهان كرفته شده است. 


\section{References}

[1] Ghaffari S, Ahmadi F, Nabavi SM, Kazem-Nezhad A. [Effects of applying hydrotherapy on quality of life in women with multiple sclerosis (Persian)]. Archives of Rehabilitation. 2008; 9(3):43-50.

[2] Bizzoco E, Lolli F, Repice AM, Hakiki B, Falcini M, Barilaro A, et al. Prevalence of neuromyelitis optica spectrum disorder and phenotype distribution. Journal of Neurology. 2009; 256(11):1891-8. doi: 10.1007/s00415-009-5171-x

[3] Olek MJ. Epidemiology, risk factors, and clinical features of multiple sclerosis. Waltham: Up To Date; 2004.

[4] Halper J. The evolution of nursing care in multiple sclerosis. International Journal of MS Care. 2000; 2(1):14-22. doi: 10.7224/1537-2073-2.1.14

[5] Seyedfatemi N, Heydari M, Hoseini AF. Self esteem and its associated factors in patients with multiple sclerosis. Iran Journal of Nursing. 2012; 25(78):14-22

[6] Engel RA, DeLuca J, Gaudino EA, Diamond BJ, Christodoulou C. Acquisition and storage deficits in multiple sclerosis. Journal of Clinical and Experimental Neuropsychology (Neuropsychology, Development and Cognition: Section A). 1998; 20(3):376-90. doi: 10.1076/jcen.20.3.376.819

[7] Peyser JM, Rao SM, LaRocca NG, Kaplan E. Guidelines for neuropsychological research in multiple sclerosis. Archives of Neurology. 1990; 47(1):94-7. doi: 10.1001/archneur.1990.00530010120030

[8] Fasoli SE, Trombly CA, Tickle Degnen L, Verfaellie MH. Effect of instructions on functional reach in persons with and without cerebrovascular accident. American Journal of Occupational Therapy. 2002; 56(4):380-90. doi: 10.5014/ajot.56.4.380

[9] Poursadoughi A, Dadkhah A, Pourmohamadreza-Tajrishi M, Biglarian A. Psycho-Rehabilitation Method (Dohsa-Hou) and Quality of Life in Children with Cerebral Palsy . Iranian Rehabilitation Journal. 2015; 13(2):28-33

[10] Bahramkhani M, Darvishi N, Keshavarz Z, Dadkhah A. [The comparison of executive functions in normal and autistic children, considering mathematics and reading abilities (Persian)]. Archives of Rehabilitation. 2013; 13:128-35.

[11] Stapleton T, Ashburn A, Stack E. A pilot study of attention deficits, balance control and falls in the subacute stage following stroke. Clinical Rehabilitation . 2001; 15(4):437-44. doi: 10.1191/026921501678310243

[12] Chiviacowsky S, Wulf G, Wally R. An external focus of attention enhances balance learning in older adults. Gait \& Posture . 2010; 32(4):572-5. doi: 10.1016/j.gaitpost.2010.08.004

[13] Dusti F, Sohrabi M, Saeidi M, Tymuri S. Effect of instructions for internal and far and near external focus of attention on balance of Multiple Sclerosis patients. Medical Journal of Mashhad University of Medical Sciences. 2013; 56(1):15-20.

[14] Shiri V, Hosseini SA, Pishyareh E, Nejati V, Biglarian A. [Study the relationship of executive functions with behavioral symptoms in children with high functioning autism (Persian)]. Archives of Rehabilitation. 2015; 16(3):208-17.
[15] Cameron MH, Poel AJ, Haselkorn JK, Linke A, Bourdette D. Falls requiring medical attention among veterans with multiple sclerosis: A cohort study. The Journal of Rehabilitation Research and Development. 2011; 48(1):13. doi: 10.1682/jrrd.2009.12.0192

[16] Bruce JM, Bruce AS, Arnett PA. Mild visual acuity disturbances are associated with performance on tests of complex visual at tention in MS. Journal of the International Neuropsychological Society. 2007; 13(03). doi: 10.1017/s1355617707070658

[17] Nobahar Ahari M, Nejati V, Hosseini SA. Attentional demands of balance under dual task conditions in young adults. Iranian Rehabilitation Journal. 2012; 10(2):66-71.

[18] Ahmadizadeh Z, Abrlahij A, Pahlevanian A. The relation between balance and sustained attention in elderly. Iranian Rehabilitation Journal. 2013; 11:65-9.

[19] Rao SM, Leo GJ, Bernardin L, Unverzagt F. Cognitive dysfunction in multiple sclerosis: I. Frequency, patterns, and prediction. Neurology. 1991; 41(5):685-91. doi: 10.1212/wnl.41.5.685

[20] Feinstein A. The clinical neuropsychiatry of multiple sclerosis. Cambridge: Cambridge University Press; 2007.

[21] Liu Ambrose T, Pang MYC, Eng JJ. Executive function is independently associated with performances of balance and mobility in community-dwelling older adults after mild stroke: Implications for falls prevention. Cerebrovascular Diseases. 2006; 23(23):203-10. doi: 10.1159/000097642

[22] D’Orio VL, Foley FW, Armentano F, Picone MA, Kim S, Holtzer R. Cognitive and motor functioning in patients with multiple sclerosis: Neuropsychological predictors of walking speed and falls. Journal of the Neurological Sciences . 2012; 316(1 2):42-6. doi: 10.1016/i.jns.2012.02.003

[23] Bonneville F, Moriarty DM, Li BS, Babb JS, Grossman RI, Gonen O. Whole-brain N-acetylaspartate concentration: correlation with $\mathrm{T} 2$-weighted lesion volume and expanded disability status scale score in cases of relapsing-remitting multiple sclerosis. American Journal of Neuroradiology. 2002; 23(3):371-5.

[24] Bogle Thorbahn LD, Newton RA. Use of the Berg Balance Test to predict falls in elderly persons. Physical Therapy. 1996; 76(6):576-83. doi: 10.1093/ptj/76.6.576

[25] Patti F. Cognitive impairment in multiple sclerosis. Multiple Sclerosis Journal . 2009; 15(1):2-8. doi: 10.1177/1352458508096684

[26] Julian LJ. Cognitive functioning in multiple sclerosis. Neurologic Clinics . 2011; 29(2):507-25. doi: 10.1016/i.ncl.2010.12.003

[27] Flavia M, Stampatori C, Zanotti D, Parrinello G, Capra R. Efficacy and specificity of intensive cognitive rehabilitation of attention and executive functions in multiple sclerosis. Journal of the Neurological Sciences . 2010; 288(1-2):101-5. doi: 10.1016/j. jns.2009.09.024

[28] MacAllister WS, Christodoulou C, Milazzo M, Preston TE Serafin D, Krupp LB, et al. Pediatric multiple sclerosis: What we know and where are we headed. Child Neuropsychology. 2013; 19(1):1-22. doi: 10.1080/09297049.2011.639758

[29] Noll KR. Predictors of quality of life in multiple sclerosis: Relationships between cognitive, physical, and subjective measures of disease burden $[\mathrm{PhD}$ dissertation]. Texas: University of Texas; 2011. 
[30] Amato MP, Zipoli V, Portaccio E. Multiple sclerosis-related cognitive changes: A review of cross-sectional and longitudinal studies. Journal of the Neurological Sciences . 2006; 245(1-2):41-6. doi: 10.1016/j.jns.2005.08.019

[31] Sailer M. Focal thinning of the cerebral cortex in multiple sclerosis. Brain. 2003; 126(8):1734 44. doi: 10.1093/brain/awg175

[32] Mainero C, Caramia F, Pozzilli C, Pisani A, Pestalozza I, Borriello $G$, et al. fMRI evidence of brain reorganization during attention and memory tasks in multiple sclerosis. NeuroImage. 2004; 21(3):858-67. doi: 10.1016/j.neuroimage.2003.10.004

[33] Achiron A. Cognitive impairment in probable multiple sclerosis. Journal of Neurology, Neurosurgery \& Psychiatry. 2003; 74(4):443-6. doi: 10.1136/jnnp.74.4.443

[34] Ruggieri RM, Palermo R, Vitello G, Gennuso M, Settipani N, Piccoli F. Cognitive impairment in patients suffering from relapsing-remitting multiple sclerosis with EDSS $<$ or $=3.5$. Acta Neurologica Scandinavica. 2003; 108(5):323-6. doi: 10.1034/j.16000404.2003.00157.x

[35] Bol Y, Duits AA, Hupperts RM, Verlinden I, Verhey FR. The impact of fatigue on cognitive functioning in patients with multiple sclerosis. Clinical Rehabilitation . 2010; 24(9):854-62. doi: $10.1177 / 0269215510367540$

[36] Rao SM, Hammeke TA, Speech TJ. Wisconsin Card Sorting Test performance in relapsing-remitting and chronic-progressive multiple sclerosis. Journal of Consulting and Clinical Psychology. 1987; 55(2):263-5. doi: 10.1037/0022-006x.55.2.263

[37] Arrondo G, Alegre M, Sepulcre J, Iriarte J, Artieda J, Villoslada P. Abnormalities in brain synchronization are correlated with cognitive impairment in multiple sclerosis. Multiple Sclerosis Journal. 2009; 15(4):509-16. doi: 10.1177/1352458508101321 
